\title{
Endogenous giant viruses shape intraspecies genomic variability in the model green alga Chlamydomonas reinhardtii
} \\ Mohammad Moniruzzaman*1 and Frank O. Aylward¹
}

Abstract:

Chlamydomonas reinhardtii is an important eukaryotic alga that has been studied as a model organism for decades. Despite extensive history as a model system, phylogenetic and genetic characteristics of viruses infecting this alga have remained elusive. We analyzed highthroughput genome sequence data of numerous $C$. reinhardtii isolates, and in six strains we discovered endogenous genomes of giant viruses reaching over several hundred kilobases in length. In addition, we have also discovered the entire genome of a closely related giant virus that is endogenized within the genome of Chlamydomonas incerta, one of the closest sequenced phylogenetic relatives of $C$. reinhardtii. Endogenous giant viruses add hundreds of new gene families to the host strains, highlighting their contribution to the pangenome dynamics and inter-strain genomic variability of $C$. reinhardtii. Our findings suggest that endogenization of giant viruses can have profound implications in shaping the population dynamics and ecology of protists in the environment.

\section{Introduction:}

Giant viruses that infect diverse eukaryotes have recently emerged as a widely distributed viral group in the biosphere $(1,2)$. These viruses belong to a broadly defined group that has been recently classified as the phylum Nucleocytoviricota (3). Although giant viruses are primarily studied as agents of mortality of diverse protists and metazoans, recent studies have demonstrated that they can drastically shape the genomes of their hosts through endogenization (4). Specifically, Giant Endogenous Viral Elements (GEVEs) that are up to several thousand kilobases long have been identified in diverse green algae (4), providing compelling evidence that endogenization of diverse giant viruses can profoundly influence host genome evolution and alter the evolutionary trajectory of host eukaryotes.

Chlamydomonas reinhardtii is a widely used model photosynthetic eukaryote with history as a model organism dating back to the 1950s (5). Here, we report that endogenous giant viruses of giant viruses endogenized in field isolates, we show that $C$. reinhardtii is a host of diverse giant viruses from distinct phylogenetic affiliations. Our results establish this widely-studied green alga as a model to study the mechanistic and evolutionary aspects of giant virus endogenization in diverse eukaryotic lineages, and provides the first insights on the genomic 44 complexity and phylogenetic history of viruses infecting $C$. reinhardtii in nature. 


\section{Results:}

We analyzed publicly available high-throughput genome sequencing data for 33 wild strains of C. reinhardtii. This data was originally generated for population genomic studies of diverse $C$. reinhardtii strains (6-8). After assembly and annotation (see Methods for details), we identified GEVEs in six of the wild strains (Figure 1B). In five of these (CC-2936, 2937, 2938, 3268, and GB-66), the GEVEs range from 315-356 Kb in size and harbored all but one Nucleocytoviricota hallmark genes, indicating that near-complete genomes of endogenous giant viruses have been retained in these strains (Figure 1B, Dataset S1). In contrast, CC-3061 harbors a GEVE 113 $\mathrm{Kb}$ in size with 5 out of the 10 hallmark genes, indicating that part of the GEVE was lost over the course of evolution (Supplementary Methods, Dataset S1). We also analyzed the assembled genome of Chlamydomonas incerta, a species phylogenetically closest to $C$. reinhardtii, for which a long-read assembled genome has been recently reported (9). This analysis revealed a GEVE $\sim 475 \mathrm{~Kb}$ long which is integrated within a single $592 \mathrm{~Kb}$ contig of $C$. incerta. (Figure 1B)

The GC-content of the C. reinhardtii GEVEs ranged from $58.27 \%$ (CC-2938) to $60.72 \%$ (CC3268 ), close to the overall genomic GC content of $C$. reinhardtii (64\%) (10). Similarly, the GC content of the $C$. incerta GEVE was $64.8 \%$, resembling the overall $G C$ content of the $C$. incerta genome (66\%) (9) (Figure 1B). The GEVEs also contained numerous spliceosomal introns, ranging from 25 (CC-3061) to 72 (C. incerta) which has been previously found to be a feature of GEVEs present in other members of the Chlorophyta (4). Together, these results suggest the GEVEs were subjected to GC-content amelioration and intron-invasion since endogenization. In addition, the GEVE in $C$. incerta was flanked by highly repetitive regions on both ends (Figure 2A). The repetitive region at the 5 '-end harbors several reverse transcriptases and transposases (Dataset S1). These regions also have higher intron density compared to the GEVE region itself, and lower number of Giant Virus Orthologous Group (GVOG) hits consistent with their eukaryotic provenance (Figure 2A). This suggests that near-complete genomes of giant viruses can integrate within highly repetitive regions of eukaryotic genomes, potentially with the facilitation of transposable elements.

Using a newly established taxonomy of Nucleocytoviricota (11), we determined the phylogenetic position of the $C$. reinhardtii and $C$. incerta GEVEs and their relationships with other chlorophyte GEVEs that were recently reported (4) (Figure 1A). Five of the strains harbored GEVEs that formed a cluster within the Imitervirales order, consistent with their high average amino acid identity. The GEVE in $C$. incerta was the closest phylogenetic relative of the Imitevirales GEVEs in $C$. reinhardtii, indicating that closely-related giant viruses infect closely related Chlamydomonas species in nature. These GEVEs formed a sister clade with the GEVEs present in six other volvocine algae and belonged to the Imitevirales family 12 (Figure 1A). These results suggest that many viruses infecting volvocine algae in nature are closely related, and provides a phylogenetic framework for future efforts on detection and isolation of viruses infecting these algal lineages. In contrast to the GEVEs that could be classified as Imitervirales, the GEVE in CC-2938 strain belonged to the Algavirales (Figure 1A), demonstrating that $C$. reinhardtii is infected by multiple phylogenetically distinct lineages of giant viruses. 
The GEVEs in C. reinhardtii encoded 99 (CC3061) to 254 (CC2937) genes, while the C. incerta GEVE encoded 355 genes. Most of the genes were shared among the Imitervirales $C$. reinhardtii GEVEs, consistent with their high average amino acid identity (AAI) to each other (Figure 1C, D). These GEVEs also shared a high number of orthogroups with the $C$. incerta GEVE. In contrast, only a few orthogroups were shared between the Imitevirales and the Algavirales GEVEs consistent with the large phylogenetic distance between these lineages. Between $\sim 44-55 \%$ of the genes in the $C$. reinhardtii and $C$. incerta GEVEs have matches to Giant Virus Orthologous Groups (GVOGs), confirming their origin in the Nucleocytoviricota (Figure 1B). In addition, different genes in these regions have best matches to giant viruses, bacteria, and eukaryotes, which is a common feature of Nucleocytoviricota members given the diverse phylogenetic origin of the genes in these viruses (12) (Figure 2A). Based on the Cluster of Orthologous Group (COG) annotations, a high proportion of the GEVE genes are involved in transcription, and DNA replication and repair; however, genes encoding translation, nucleotide metabolism and transport, signal transduction, and posttranslational modification were also present, consistent with the diverse functional potential encoded by numerous Nucleocytoviricota (Figure 2B).

A previous study has shown that several field strains of $C$. reinhardtii harbor many genes that are absent in the reference genome (7), which were possibly acquired from diverse sources. To quantify the amount of novel genetic material contributed by giant viruses to $C$. reinhardtii, we estimated the number of unique gene families in the analyzed $C$. reinhardtii field strains that are absent in the reference strain CC-503. On average $~ 2.24 \%$ of the genes in the field strains were unique compared to the reference strain (Figure $2 \mathrm{C}$ ). Moreover, the GEVE-harboring field strains have significantly more unique genes compared to those without GEVEs, (Two-sided Man-Whitney U-test $p$-value $<0.05$ ). These results suggest that endogenization of giant viruses is an important component of inter-strain variability in C. reinhardtii. Recent studies have highlighted the importance of horizontal gene transfer (HGT) in structuring the pangenome of diverse eukaryotes $(13,14)$, and genes originating from endogenous Nucleocytoviricota were found to shape the genomes of many algal lineages, including members of the Chlorophyta (4, 15). Compared to the GEVE-free strains, GEVE-containing strains harbored a significantly higher proportion of genes from several COG categories including Transcription, Signal Transduction, and Replication and Repair (Two sided Mann-Whitney $U$ test $p$-value $<0.05$ ) (Figure $2 \mathrm{C}$ ). All together, these GEVEs contributed many genes with known functions, including glycosyltransferases, proteins involved in DNA repair, oxidative stress, and heat shock regulation (Dataset S1).

\section{Discussion:}

While much work remains to elucidate the role of GEVEs in shaping the ecological dynamics of C. reinhardtii, several possibilities remain open. Some of the genes contributed by the GEVEs can be potentially co-opted by the host, leading to changes in certain phenotypes compared to closely related strains without GEVEs. Strain-specific endogenization can also potentially lead to intraspecific variations in chromosome structure, partly mediated by the GEVE-encoded mobile elements (16). Finally, it is also possible that some of these GEVE-loci can produce 
siRNAs that might participate in antiviral defense, and similar phenomena has been suggested for the virus-like loci in the genome of moss (Physcomitrella patens) (17). Recent studies on large-scale endogenization of giant virus genomes in diverse green algae and patchwork of viral genes in many algal lineages suggest that interactions between giant viruses and their algal hosts frequently shape the host genomes $(4,15)$, and therefore represents a major research frontier for studying the effect of giant viruses in influencing the (HGT) landscape. Our results indicate that endogenization of giant viruses can lead to large-scale genomic variability not only between algal species, but also between strains within the same population. Results reported in this study therefore represent an important advance in our understanding of how giant viruses shape the genome evolution of their hosts, while also expanding the scope of $C$. reinhardtii as a model organism to study the evolutionary fate and consequences of giant virus endogenization.

\section{Methods:}

All methods and relevant citations are available in the 'Supplementary Information' file.

\section{Data and Code availability:}

Dataset $\mathrm{S} 1$ contains information regarding the raw data source, GEVE functional annotations, hallmark gene distribution in each GEVE and coverage information of the partial GEVE in CC3061. Dataset S2 contains figures related to the Method section - TNF dendrogram for the CC2938 GEVE and promer alignment plots between C. reinhardtii Imitevirales GEVEs and C. incerta GEVE.

All the GEVE fasta files, unique gene fasta in each of the strains and their annotations, and concatenated alignment file used to build the phylogenetic tree in Figure 1 are available in Zenodo: https://zenodo.org/record/4958215

Code and instructions for ViralRecall v2.0 and NCLDV marker search scripts are available at: github.com/faylward.

\section{Acknowledgement}

We acknowledge the use of the Virginia Tech Advanced Research Computing Center for bioinformatic analyses performed in this study. This work was supported by grants from the Institute for Critical Technology and Applied Science and the NSF (IIBR-1918271) and a Simons Early Career Award in Marine Microbial Ecology and Evolution to F.O.A.

\section{Conflict of interest statement}

The authors declare no conflict of interest relevant to the content of the manuscript. 
177 Figure 1: General features and phylogeny of the GEVEs. A) Maximum likelihood

178 phylogenetic tree of the GEVEs and representative members from diverse NCLDV families

179 constructed from a concatenated alignment of seven NCLDV hallmark genes (see Methods).

180 Individual families within each order are indicated with abbreviations (IM - Imitevirale, AG -

181 Algavirales) followed by family numbers, as specified in Aylward et al, 2019 (11). IDs of the

182 GEVEs are indicated in bold-italic. B) Basic statistics of the GEVEs present in various field

183 strains of $C$. reinhardtii and the GEVE present in the C. incerta genome. C) Heatmap

184 representing the number of orthologous groups of proteins shared across all the GEVEs. D)

185 Heatmap representing the average amino acid (AAI) \% among the GEVEs.

186 * Length includes the eukaryotic regions flanking the $C$. incerta GEVE.

Figure 2: GEVE genomic and functional characteristics. A) Circular plots of two representative GEVEs in $C$. reinhardtii and the GEVE present in $C$. incerta. For $C$. reinhardtii one representative Imitevirales GEVE (CC-2937) and the Algavirales GEVE (CC-2938) are shown. Circle plots show Giant Virus Orthologous Group (GVOG) hidden Markov model (HMM) hits, spliceosomal introns and the best LAST hit matches (see Supplementary Methods). Internal blue links delineate the duplicated regions. The eukaryotic regions flanking the $C$. incerta GEVE are delineated with light blue stripes. B) Functional potential encoded in the GEVEs as EggNOG categories. Proportion of genes in each category is normalized across all the NOG categories except category S (function unknown) and R (general function prediction). Raw functional annotations are present in Dataset S1. C) Unique genes in the field strains of $C$. reinhardtii compared to the reference strain CC-503. The heatmap represents \% of unique genes that can be classified in different EggNOG categories (except category [R] - genel function prediction and [S] - function unknown). Categories marked with '**' are significantly overrepresented in the GEVE-containing strains compared to those without GEVEs. The bar plot on top of the heatmap represents \% of unique genes in each strain. The names of the abbreviated categories are the same as in figure 2B. GEVE-containing strains have significantly higher percentages of unique genes compared to the strains without GEVEs.

\section{References:}

1. M. Moniruzzaman, C. A. Martinez-Gutierrez, A. R. Weinheimer, F. O. Aylward, Dynamic genome evolution and complex virocell metabolism of globally-distributed giant viruses. Nat. Commun. 11, 1710 (2020).

2. H. Endo, et al., Biogeography of marine giant viruses reveals their interplay with eukaryotes and ecological functions. Nat Ecol Evol 4, 1639-1649 (2020).

3. E. V. Koonin, et al., Global Organization and Proposed Megataxonomy of the Virus World. Microbiol. Mol. Biol. Rev. 84 (2020).

4. M. Moniruzzaman, A. R. Weinheimer, C. A. Martinez-Gutierrez, F. O. Aylward, Widespread endogenization of giant viruses shapes genomes of green algae. Nature (2020) https:/doi.org/10.1038/s41586-020-2924-2.

5. S. Sasso, H. Stibor, M. Mittag, A. R. Grossman, From molecular manipulation of domesticated to survival in nature. Elife 7 (2018). 
6. R. J. Craig, et al., Patterns of population structure and complex haplotype sharing among field isolates of the green alga Chlamydomonas reinhardtii. Mol. Ecol. 28, 3977-3993 (2019).

7. J. M. Flowers, et al., Whole-Genome Resequencing Reveals Extensive Natural Variation in the Model Green Alga Chlamydomonas reinhardtii. Plant Cell 27, 2353-2369 (2015).

8. A. R. Hasan, J. K. Duggal, R. W. Ness, Consequences of recombination for the evolution of the mating type locus in Chlamydomonas reinhardtii. New Phytol. 224, 1339-1348 (2019).

9. R. J. Craig, A. R. Hasan, R. W. Ness, P. D. Keightley, Comparative genomics of

14. S. J. Sibbald, L. Eme, J. M. Archibald, A. J. Roger, Lateral Gene Transfer Mechanisms and

15. D. R. Nelson, et al., Large-scale genome sequencing reveals the driving forces of viruses in microalgal evolution. Cell Host Microbe 29, 250-266.e8 (2021).

16. J. Filée, Giant viruses and their mobile genetic elements: the molecular symbiosis hypothesis. Curr. Opin. Virol. 33, 81-88 (2018).

17. D. Lang, et al., The Physcomitrella patens chromosome-scale assembly reveals moss genome structure and evolution. Plant J. 93, 515-533 (2018). 
bioRxiv preprint doi: https://doi.org/10.1101/2021.11.30.470594: this version posted November 30, 2021. The copyright holder for this preprint (which was not certified by peer review) is the author/funder, who has granted bioRxiv a license to display the preprint in perpetuity. It is made available under aCC-BY-NC 4.0 International license. 
A)

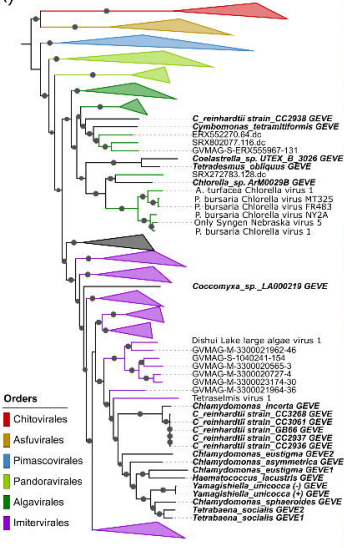

B)

\begin{tabular}{|c|c|c|c|c|c|c|c|}
\hline \multirow{4}{*}{$\begin{array}{l}\text { AG-04 } \\
\text { AG-01 }\end{array}$} & Strain ID & $\begin{array}{l}\text { GEVE } \\
\text { Length } \\
\text { (Kbp) }\end{array}$ & $\begin{array}{l}\text { Contig } \\
\text { Count }\end{array}$ & $\begin{array}{c}\text { Protein } \\
\text { count }\end{array}$ & $\begin{array}{l}\text { Intron } \\
\text { count }\end{array}$ & GC\% & $\begin{array}{c}\% \text { of } \\
\text { GVOG } \\
\text { hits }\end{array}$ \\
\hline & $c c-3061$ & 112.7 & 8 & 99 & 25 & 60.51 & 56 \\
\hline & $c c-2938$ & 315.2 & 11 & 214 & 43 & 58.27 & 44 \\
\hline & GB-EG & 325.7 & 23 & 252 & 60 & 59.24 & 50 \\
\hline \multirow{4}{*}{$A G-03$} & $C C-3268$ & 333.8 & 8 & 242 & 52 & 60.72 & 51 \\
\hline & CC-2936 & 335.5 & 11 & 245 & 55 & 60.32 & 52 \\
\hline & CC-2937 & 356.0 & 18 & 254 & 57 & 60.51 & 50 \\
\hline & C. incerta & $592.1^{8}$ & 1 & 355 & 72 & 64.78 & 48 \\
\hline
\end{tabular}

AG-02

C)

IM- 02,18

IM-14

IM-16

IM-13

IM-06

IM-07

IM-12

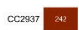

Number of orthogroups

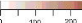

$$
\mathrm{CC} 2938 \quad 10 \quad 10
$$$$
\int_{0}^{100}+20
$$

$$
\text { C03061 a7 } \quad \text { ar } 7
$$$$
\text { Cᄃ3268 248 } 220
$$$$
\text { sBo6 }
$$

C. ninoerts

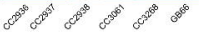

D)
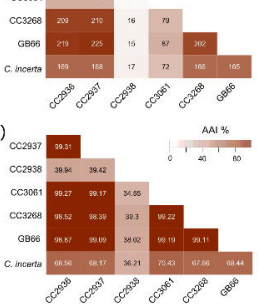

Tree scale: 1 
A)

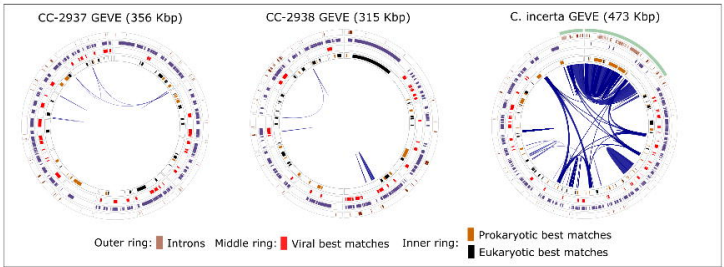

B)

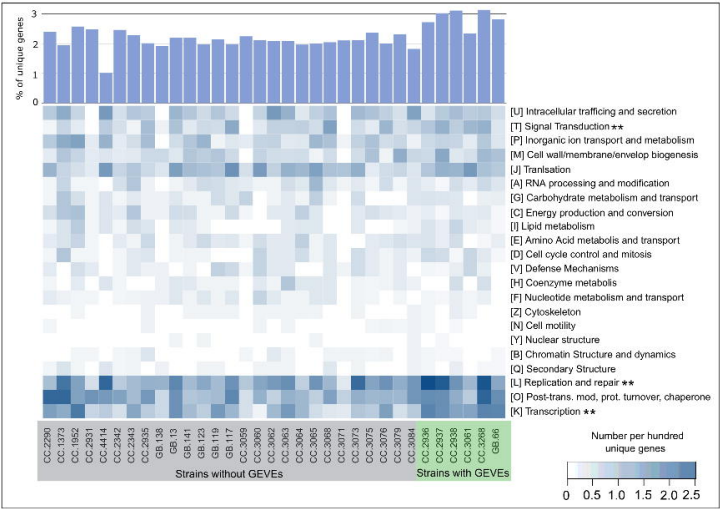

